\title{
Anti-Warburg effect of rosmarinic acid via miR-I55 in gastric cancer cells
}

\author{
This article was published in the following Dove Press journal: \\ Drug Design, Development and Therapy \\ 19 May 2015 \\ Number of times this article has been viewed
}

\section{Shuai Han* \\ Shaohua Yang* \\ Zhai Cai \\ Dongyue Pan \\ Zhou Li \\ Zonghai Huang \\ Pusheng Zhang \\ Huijuan Zhu \\ Lijun Lei \\ Weiwei Wang}

Department of General Surgery, Zhujiang Hospital, Southern Medical University, Guangzhou, People's

Republic of China

*These authors contributed equally to this study
Correspondence: Zhou Li

Department of General Surgery, Zhujiang Hospital, Southern Medical University

Guangzhou, Guangzhou 510282,

People's Republic of China

$\mathrm{Tel} / \mathrm{fax}+862062782397$

Email leezhou888@yeah.net
Background: The Warburg effect refers to glycolytic production of adenosine triphosphate under aerobic conditions, and is a universal property of most cancer cells. Chronic inflammation is a key factor promoting the Warburg effect. This study aimed to determine whether rosmarinic acid (RA) has an anti-Warburg effect in gastric carcinoma in vitro and in vivo. The mechanism for the anti-Warburg effect was also investigated.

Methods: An MTT assay was used to examine MKN45 cell growth in vitro. An enzyme-linked immunosorbent assay was used to detect proinflammatory cytokines. Real-time polymerase chain reaction was used to evaluate levels of microRNA expression in cells. Protein expression was determined by Western blotting assay. Mouse xenograft models were established using MKN45 cells to assess the anti-Warburg effect in gastric carcinoma in vivo.

Results: RA suppressed glucose uptake and lactate production. It also inhibited expression of transcription factor hypoxia-inducible factor- $1 \alpha$, which affects the glycolytic pathway. Inflammation promoted the Warburg effect in cancer cells. As expected, RA inhibited proinflammatory cytokines and microRNAs related to inflammation, suggesting that RA may suppress the Warburg effect via an inflammatory pathway, such as that involving interleukin (IL)-6/signal transducer and activator of transcription-3 (STAT3). miR-155 was found to be an important mediator in the relationship between inflammation and tumorigenesis. We further showed that miR-155 was the target gene regulating the Warburg effect via inactivation of the IL-6/STAT3 pathway. Moreover, we found that RA suppressed the Warburg effect in vivo.

Conclusion: RA might potentially be a therapeutic agent for suppressing the Warburg effect in gastric carcinoma.

Keywords: rosmarinic acid, miR-155, inflammation, Warburg effect, MKN45

\section{Introduction}

In the 1920s, Otto Warburg reported that cancer cells produce most of their energy via the glycolytic pathway under aerobic conditions. ${ }^{1}$ This phenomenon, which became known as the Warburg effect, is a feature of most cancer cells, ${ }^{2}$ and tumor cells must adapt to these adverse conditions until new blood vessels are formed and oxygen tension returns to normal. ${ }^{3}$ Consequently, tumor cells have to change their metabolic pathway, upregulating the glycolytic pathway through induction of hypoxia-inducible factor-1 (HIF-1). ${ }^{4}$ HIF-1 is a heterodimer, consisting of a stably expressed HIF-1 $\beta$ subunit and an unstable HIF-1 $\alpha$ subunit. The amount of HIF- $1 \alpha$ in cells is regulated and determined by the relative rates of its synthesis and degradation. Synthesis of HIF- $1 \alpha$ is modulated by an oxygen-independent mechanism, whereas its degradation occurs via an oxygen-dependent mechanism. Under hypoxic conditions, HIF-1 $\alpha$ is present at very low levels due to rapid degradation. ${ }^{5}$

Diseases such as allergy, asthma, diabetes, and cancer can be triggered by the inflammatory response. ${ }^{6,7}$ Chronic inflammation is believed to be a key factor promoting 
tumorigenesis, and the link between inflammation and cancer is supported by recent clinical studies. ${ }^{8-10}$ The Warburg effect is a hallmark of carcinoma, and proinflammatory cytokines relevant to carcinoma are associated with cell survival, proliferation, and angiogenesis. ${ }^{11}$ Interleukin (IL)-6 acts as a proinflammatory cytokine. The classic IL-6 pathway plays a vital role not only in normal developmental processes and tissue homeostasis, but also in acute phase immune responses, inflammatory disease, and development of cancer. Another proinflammatory cytokine, tumor necrosis factor alpha (TNF- $\alpha$ ), is also involved in the microenvironment of tumorigenesis. Low levels of TNF- $\alpha$ can dramatically increase reliance on glycolysis, export of lactate, and expression of the glucose transporter. ${ }^{12}$

Rosmarinic acid (RA) is isolated from herbal balm mint plants, such as Rosmarinus officinalis, Melissa officinalis, and Prunella vulgaris L. ${ }^{13,14}$ It is a naturally occurring hydroxylated compound with anti-inflammatory, anticancer, antimutagenic, antibacterial, and antiviral activity. ${ }^{15-17}$ However, the effects of RA on the Warburg effect have not been previously addressed.

In the present study, we demonstrated that RA inhibits proliferation of MKN45 human gastric cancer cells and the Warburg effect in vitro. A possible molecular mechanism that could underlie these behaviors was sought.

\section{Materials and methods Materials}

RA was purchased from Yuanye Biotechnology (Shanghai, People's Republic of China) and its purity was assessed by high-performance liquid chromatography as being $>98 \%$. The structure of the compound is shown in Figure 1A. The 3-(4,5-dimethylthiazol-2-yl)-2,5-diphenyltetrazolium bromide (MTT) and 5-bromo-4-chloro-3-indolyl-phosphate/ nitro blue tetrazolium were purchased from Sigma-Aldrich Co. (St Louis, MO, USA). Human signal transducer and activator of transcription-3 (STAT3) antibody was obtained from Abcam (Cambridge, MA, USA), human HIF-1 $\alpha$ antibody was obtained from Novus Biologicals (Littleton, CO, USA), and human p-STAT3 antibody was obtained from Cell Signaling (Beverly, MA, USA).

\section{Cells and animals}

MKN45 human gastric cancer cells were purchased from the Cell Bank of Type Culture Collection of Chinese Academy of Sciences (Shanghai, People's Republic of China). MKN45 cells were cultured in Roswell Park Memorial
A
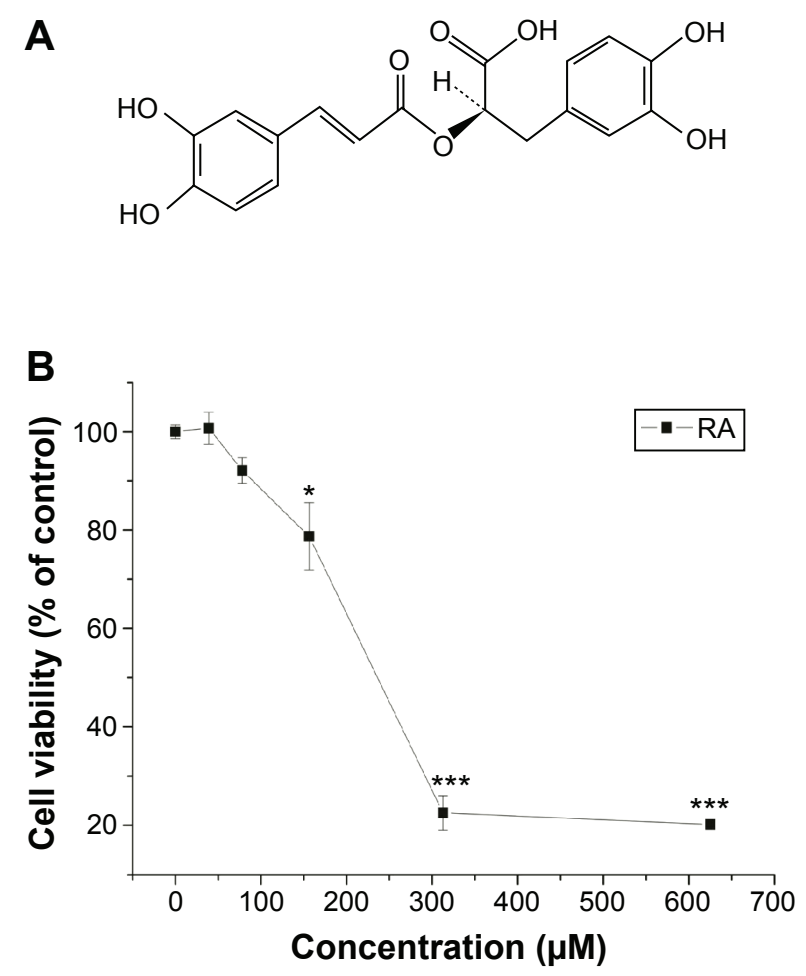

Figure I Characteristics of RA.

Notes: (A) Structure of RA: [4aR-(4aa,9b, 10a, 10ab)]-1,3,4,9,10, 10a-hexahydro5,6,9-trihydroxy-I, I-dimethyl-7-(I-methylethyl)-2H-10,4a-(epoxymethano) phenanthren-12-one. (B) Cytotoxic effect of RA on MKN45 cells. The data are presented as the mean \pm standard deviation. Significant differences from control are indicated by $* P<0.05 ; * * * P<0.001$.

Abbreviation: RA, rosmarinic acid.

Institute (RPMI) 1640 medium supplemented with $10 \%$ heat-inactivated bovine serum, penicillin $100 \mathrm{U} / \mathrm{mL}$, and streptomycin $100 \mathrm{U} / \mathrm{mL}$. Cells were incubated at $37^{\circ} \mathrm{C}$ in a humidified atmosphere of $95 \%$ air and $5 \% \mathrm{CO}_{2}$. Male BALB/c-nu mice (aged 5 weeks, license number 44008500006317) were obtained from the Animal Center of Zhongshan University and maintained on standard chow and water. All experiments were carried out with the approval of the administrative panel at the Laboratory Animal Care of Southern Medical University.

\section{Cytotoxicity assay}

The cytotoxicity assay was performed using the MTT method. Cells were placed in $96-$ well culture plates $\left(1 \times 10^{5}\right.$ cells per well) and allowed to attach for 12 hours before treatment. The cells were treated with RA at concentrations ranging from 0 to $600 \mu \mathrm{M}$. Cell viability was evaluated by MTT assay after 24 hours of treatment. The optical density in the control and drug-treated wells was measured at a wavelength of $570 \mathrm{~nm}$. The cytotoxicity of RA was expressed as the $\mathrm{IC}_{50}$ 
(concentration of $50 \%$ cytotoxicity), which was extrapolated from linear regression analysis of the experimental data.

\section{Measurement of glucose uptake}

Twenty-four hours before the assay, the cells were counted and $2.0 \times 10^{5}$ cells were incubated in $1 \mathrm{~mL}$ of RPMI 1640 medium containing $10 \%$ fetal bovine serum. The cells were then treated with RA $\left(\mathrm{IC}_{50}, 1 / 2 \mathrm{IC}_{50}, 1 / 4 \mathrm{IC}_{50}, 0\right)$ for 24 hours. After 24 hours, the supernatant was removed and fresh RPMI 1640 medium without fetal bovine serum was added. The glucose concentration in samples with the same medium was determined at different time points ( $0,1,2,3,4$ hours) using a glucose assay kit (Nanjing Jiancheng Bioengineering Institute, Nanjing, People's Republic of China) according to the manufacturer's instructions. Optical density was measured with a spectrophotometer at $505 \mathrm{~nm}$.

Absorption spectra for the cultures were obtained from actively growing late phase cultures with dilution to a density of about 0.2 at the nominally robust wavelength using a sensitive spectrophotometer. The specific methods used for the spectrophotometer assay were performed according to a previous study. ${ }^{18}$

\section{Lactate measurement}

Twenty-four hours before the lactate assay, cells were counted and $2.0 \times 10^{5}$ cells were incubated in $1 \mathrm{~mL}$ of RPMI 1640 medium containing $10 \%$ fetal bovine serum. The cells were then treated with RA $\left(\mathrm{IC}_{50}, 1 / 2 \mathrm{IC}_{50}, 1 / 4 \mathrm{IC}_{50}, 0\right)$ for 24 hours. After 24 hours, the supernatant was removed and fresh RPMI 1640 medium without fetal bovine serum was added. The lactate concentration in samples in the same medium was determined at each time point $(0,1,2,3,4$ hours $)$ using a lactate assay colorimetric kit (Nanjing Jiancheng Bioengineering Institute) according to the manufacturer's instructions. Optical density was measured with a spectrophotometer at $530 \mathrm{~nm}$.

\section{Enzyme-linked immunosorbent assay for IL-6, IL-I $\beta$, TNF- $\alpha$, and TNFsR-I}

Proinflammatory cytokines are believed to contribute to tumorigenesis. Production of cytokines (IL-6, IL-1 $\beta$, TNF- $\alpha$, and soluble tumor necrosis factor receptor 1 [TNFsR-1]) was measured using a standard sandwich enzyme-linked immunosorbent assay to assess the pharmaceutical effect of cytokines on cytokine quantity. Cytokine quantities in the samples were calculated from standard curves of recombinant cytokines using a regression linear method. The assay was performed according to the instructions supplied by the manufacturer (Yuanye Biotechnology). Absorbance results were obtained using a spectrophotometer.

\section{RNA extraction and quantification}

MicroRNA (miRNA) was extracted using an miRNA rapid extraction kit (Bioteke Corporation, Beijing, People's Republic of China) according to the manufacturer's instructions. Reverse transcription was performed using an miRNA cDNA synthesis kit (Bioteke Corporation). Realtime polymerase chain reaction (PCR) was performed using an miRNA quantitative PCR detection kit (SYBR Green, Bioteke Corporation) with an iCycler1 thermal cycler (Bio-Rad, Hercules, CA, USA). U6 RNA was used as an miRNA internal control. The primers used for real-time PCR are listed in Table 1.

\section{Transfection by miRNA agomir}

Cells seeded at $1.0 \times 10^{5}$ per well in 24-well cell culture plates were transfected with $50 \mathrm{nM}$ hsa-miR-155-5p agomir from Biotend Biological Technology (Shanghai, People's Republic of China) using Lipofectamine ${ }^{\mathrm{TM}} 2000$ (Invitrogen, Carlsbad, CA, USA) according to the supplier's instructions. The efficiency of miRNA agomir was then analyzed using quantitative real-time PCR.

\section{Western blotting}

After cells were treated with or without RA at various concentrations for 24 hours, total proteins were isolated using previously described methods. ${ }^{18}$ Equal amounts of protein were size-fractionated by $10 \%$ sodium dodecyl sulfatepolyacrylamide gel electrophoresis and electrotransferred to nitrocellulose membranes. The membranes were incubated overnight with anti-STAT3, anti-p-STAT3, and HIF-1 $\alpha$ antibodies diluted to 1:1,000, and the bound antibodies were visualized with 5-bromo-4-chloro-3-indolyl-phosphate/nitro blue tetrazolium substrate reagent.

Table I Primer sequences for quantitative polymerase chain reaction analysis

\begin{tabular}{ll}
\hline Gene name & Primer $\left(\mathbf{5}^{\prime} \rightarrow \mathbf{3}^{\prime}\right)$ \\
\hline hsa-miR-I55-5p & GGGAATGCTAATCGTGATAGGGGT \\
hsa-miR-155-3p & GGGCTCCTACATATTGGCATTAACA \\
hsa-miR-I $46 a-5 p$ & GGGTGAGAACTGAATTCCATGGGTT \\
hsa-miR-223-3p & GGTGTCAGTTTGTCAAATACCCCAG \\
hsa-miR-223-5p & GGGCGTGTATTTGACAAGCTGAGTT \\
U6 & GGATGACACGCAAATTCGTGAAA \\
\hline
\end{tabular}




\section{Animal experiments}

For subcutaneous implantation, $5 \times 10^{6}$ MKN45 cells suspended in $100 \mu \mathrm{L}$ of phosphate-buffered saline were injected subcutaneously into the flank of BALB/c-nu mice. ${ }^{19}$ Tumor-bearing mice were randomly divided into two groups, with each group consisting of five mice. The dosage of RA used in the mice had been investigated in a previous study, so a dosage of $2 \mathrm{mg} / \mathrm{kg}$ was defined as the optimal concentration (data not shown). Tumor-bearing mice were treated with RA via celiac injection at the dosage of $2 \mathrm{mg} / \mathrm{kg}$ for 14 days. The mice were then euthanized and examined 2 weeks after subcutaneous implantation. Lactate generation and glucose uptake was evaluated. All experiments involving animals were approved by The Ethics Committee of the Southern Medical University. The animal experiment was conducted in accordance with national laws on animal welfare in the People's Republic of China.

\section{Statistical analysis}

The data are shown as the mean \pm standard deviation. The statistical analysis was performed using Origin version 7.5 software to evaluate the significance of differences between groups, and the significance was considered to be $P<0.05, P<0.01$, and $P<0.001$. All data points represent the mean of triplicates.

\section{Results \\ Cytotoxicity effect of RA}

To assess the anticancer effect of RA, an MTT assay was performed for MKN45 cell growth. The cytotoxic effect of RA on MKN45 cell growth is shown in Figure 1. The data indicate that the cytotoxicity of RA was dose-dependent and showed a different sensitivity to the inhibitory effect of the RA. The results show that RA significantly decreased cell viability, and the $\mathrm{IC}_{50}$ for MKN45 cells was $240.2 \mu \mathrm{M}$. In brief, RA significantly inhibited MKN45 cell growth.

\section{Effect of RA on glucose consumption, lactate generation, and HIF-I $\alpha$}

Glucose consumption and lactate generation are central to the Warburg effect. Increased glucose uptake in cancer cells and the physiological consequence of increased lactate production were first described by Warburg et al. ${ }^{20}$ The effect of RA on glucose consumption is shown in Figure 2A. A graded decrease in glucose consumption was observed with increasing doses of RA. To evaluate lactate generation in RA-treated MKN45 cells, we detected lactate generation at the time points of $0,1,2,3$, and 4 hours after 24 hours of treatment with RA. The effect of RA on lactate generation is shown in Figure 2B, suggesting that RA has the ability to inhibit lactate generation. Hypoxia has long been related

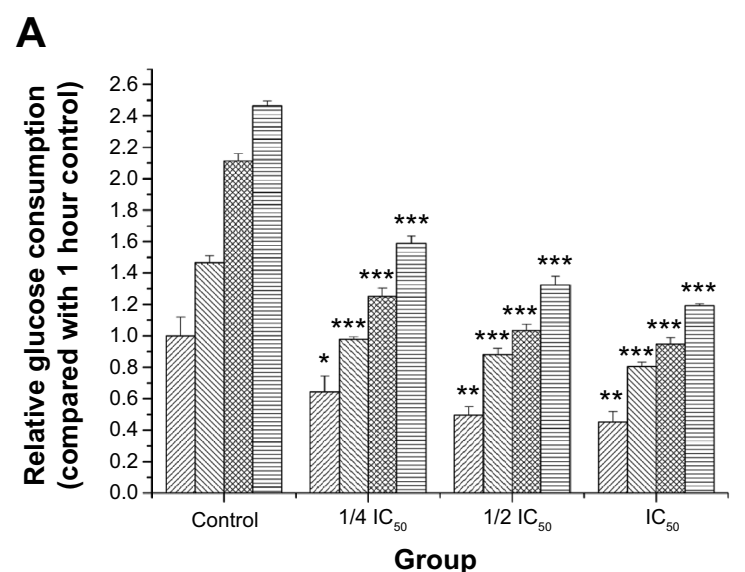

B

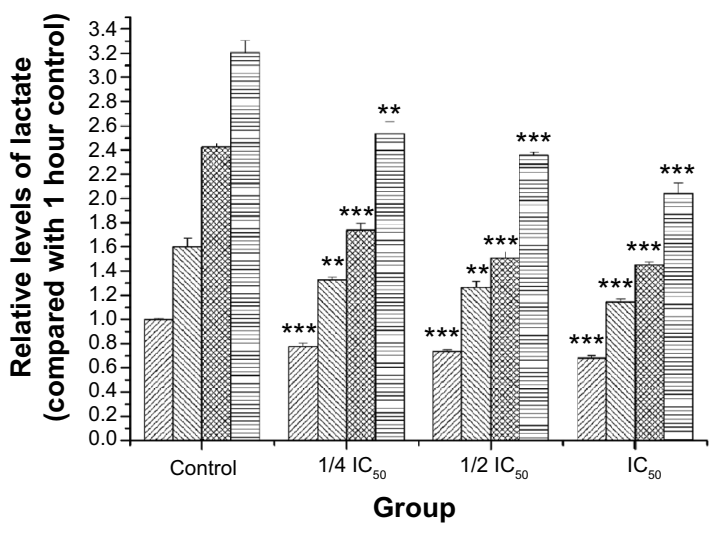

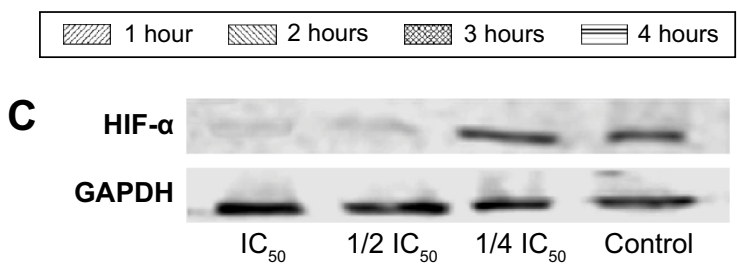

Figure 2 Glucose consumption, lactate generation, and HIF-I $\alpha$ levels after treatment with RA.

Notes: (A) Relative glucose consumption levels at I, 2, 3, and 4 hours after 24 hours of treatment with RA. (B) Relative lactate generation levels at I, 2, 3, and 4 hours after 24 hours of treatment with RA. (C) Effect of RA on transcription factor HIF-I $\alpha$. The data are presented as the mean \pm standard deviation. Significant differences from control are indicated by $* P<0.05$; $* * P<0.01$; $* * * P<0.001$.

Abbreviations: RA, rosmarinic acid; HIF-I $\alpha$, hypoxia-inducible factor-I alpha; GAPDH, glyceraldehyde-3-phosphate dehydrogenase; IC ${ }_{50}$, concentration of $50 \%$ cytotoxicity. 
to the Warburg effect. It has been shown that activation of HIF- $1 \alpha$ contributes to the Warburg effect under hypoxic conditions by coordinated upregulation of glycolysis and downregulation of oxidative phosphorylation. Figure $2 \mathrm{C}$ shows that RA suppressed expression of HIF-1 $\alpha$, which plays a vital role in the Warburg effect. Therefore, RA strongly inhibited the Warburg effect.

\section{Effects of RA on cytokine levels}

According to a previous report, ${ }^{21}$ chronic inflammation is a key factor promoting tumorigenesis, so we examined levels of cytokines linked to inflammation by enzyme-linked immunosorbent assay. Figure 3A shows that levels of cytokines linked to inflammation were suppressed in a dose-dependent manner, and significantly so for IL-6.

\section{Effects of RA on miRNA expression- related inflammation}

miR-155, miR-223, and miR-146a are negative regulators of inflammation. ${ }^{22}$ Therefore, miRNA expression related to inflammation was evaluated by real-time PCR. Figure 3B shows that RA suppressed miRNA expression-related inflammation in a dose-dependent manner, with the exception of miR-223. Expression of miRNA-155-5p RA was significantly inhibited by RA.

\section{Effects of RA on STAT3 transcription factor}

To further characterize the IL-6-STAT3 pathway activated by RA, we determined STAT3 transcription factor and STAT3 phosphorylation levels. STAT3 and p-STAT3 protein levels are shown in Figure 3C. The results indicate that RA not only had the ability to inhibit expression of STAT3 transcription factor, but could also suppress phosphorylation of STAT3.

\section{Effect of RA on miR-I55-5p expression after intervention with miR-I55-5p agomir}

After finding that RA could significantly inhibit expression of miRNA-155-5p, we investigated whether miRNA-155-5p has a key role in regulating the Warburg effect. After intervention
A

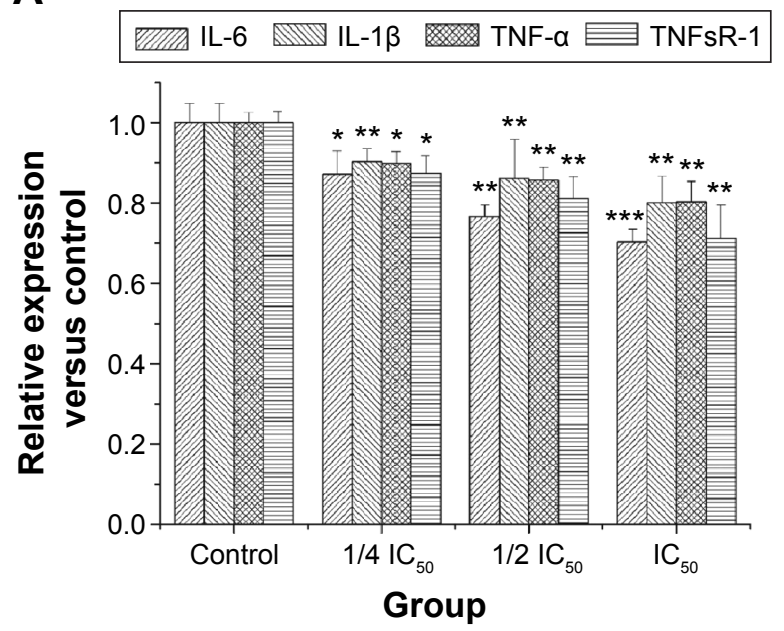

B

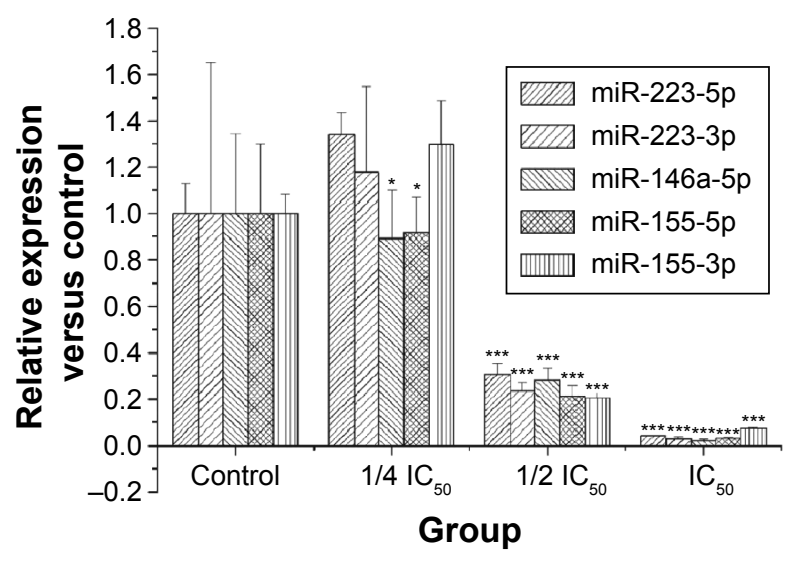

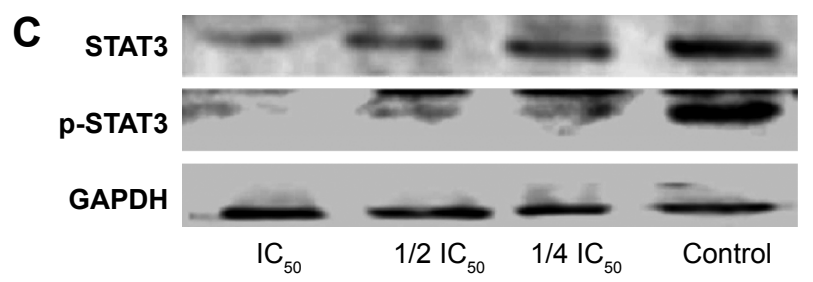

Figure 3 Effects of RA on cytokines, miRNA, STAT3, and p-STAT3 levels.

Notes: (A) Effect of RA on cytokine levels. The inhibitory effects of RA $\left(0, I C_{50}, I / 2 I C_{50}\right.$, and I/4 IC $\left.C_{50}\right)$ against IL-6, IL-I $\beta$, TNF- $\alpha$, and TNFsR-I were examined according to enzyme-linked immunosorbent assay. (B) Effect of RA on miRNA expression levels. miRNA expression levels were calculated by the target miRNA/U6 expression ratio. (C) Effect of RA on levels of STAT3 and p-STAT3. The data are presented as the mean \pm standard deviation. Significant differences from control are indicated by $* P<0.05$; $* * P<0.01$; ***P $<0.001$.

Abbreviations: RA, rosmarinic acid; GAPDH, glyceraldehyde-3-phosphate dehydrogenase; miRNA, microRNA; STAT3, signal transducer and activator of transcription-3; p-STAT3, phosphorylated STAT3; IL, interleukin; TNF- $\alpha$, tumor necrosis factor-alpha; TNFsR-I, soluble tumor necrosis factor receptor I; IC ${ }_{50}$, concentration of $50 \%$ cytotoxicity. 
with miR-155-5p agomir, RA still strongly inhibited expression of miRNA-155-5p (Figure 4A).

\section{Effect of RA on glucose consumption, lactate generation, and HIF-I $\alpha$ after intervention with miR-I55-5p agomir}

We detected glucose consumption and lactate generation at the time point of 0 and 3 hours after 24 hours of treatment with RA. The effect of RA on glucose consumption and lactate generation is shown in Figure 4B and C. Overexpression of miRNA-155-5p resulted in higher glucose consumption and more lactate generation. However, RA had the ability to reverse this phenomenon. Western blotting was used to evaluate whether RA could reverse the elevation of HIF- $1 \alpha$. Figure 4D shows that RA suppressed the expression of HIF-1 $\alpha$ after intervention with miR-155-5p agomir.

\section{Effect of RA on IL-6/STAT3 pathway after intervention with miR-I55-5p agomir}

IL-6 was inhibited by RA after intervention with miR-155-5p agomir (Figure 4E). STAT3 and p-STAT3 protein levels after intervention with miR-155-5p agomir are shown in Figure 4F. These results indicate that RA not only had the ability to inhibit expression of STAT3 transcription factor, but also had the ability to suppress phosphorylation of STAT3, suggesting that RA may affect the IL-6/STAT3 pathway via miR-155.

\section{Anti-Warburg effect of RA in vivo}

When solid tumors in BALB/c-nu mice were treated with RA, suppression of tumor growth was observed. When tumorbearing mice were treated with $2 \mathrm{mg} / \mathrm{kg}$ of RA for 14 days, there was no loss of body weight in the mice when compared with the control group during the whole treatment period. The effect of RA on glucose consumption, lactate generation, and the Warburg effect is shown in Figure 5. The results demonstrate that RA could suppress the Warburg effect in vivo, and a dose of $2 \mathrm{mg} / \mathrm{kg}$ can be recommended.

\section{Discussion}

Gastric cancer is one of the most common malignant carcinomas. Altered metabolism inducing a change in the microenvironment is a common feature of most cancer cells. ${ }^{23,24}$ HIF-1 plays an important role in glucose metabolism in cancer cells. The presence of HIF-1 can enhance the expression of several enzymes related to glycolysis, including hexokinase, phosphofructokinase, and pyruvate kinase-1. Therefore, inhibition of HIF-1 may be a vital and effective strategy for anticancer therapy. ${ }^{25}$ Our results indicate that RA is also cytotoxic, suggesting an anticancer effect. Further, the cytotoxicity of RA was dose-dependent, which is consistent with a previous report. ${ }^{26}$ Increased glucose uptake in cancer cells and the physiological consequences of increased lactate production were first reported by Warburg et al. ${ }^{20}$ Our study also indicates that RA has a strong ability to suppress glucose consumption and lactate generation. Further, RA had a strong inhibitory effect on HIF-1 $\alpha$, a key transcription factor in the Warburg effect. RA also had an inhibitory effect on the Warburg effect in vivo. Above all, the data demonstrate that the RA effectively inhibits the Warburg effect.

Inappropriate triggering of the inflammatory response contributes to development of cancer. Proinflammatory cytokines including IL- 6 , IL-1 $\beta$, TNF- $\alpha$, and certain growth factors relevant to tumorigenesis are potent STAT3 activators, while a number of genes associated with cell survival, proliferation, and angiogenesis are downstream targets of STAT $3 .{ }^{8}$ The present study investigated the effects of RA on levels of cytokines, including IL-6, IL-1 $\beta$, TNF- $\alpha$, and TNFsR-1. The results indicate that all of these cytokines, which are linked to inflammation, were suppressed in a dose-dependent manner. It has been reported that STAT3 is overexpressed in solid tumors, and is associated with tumorigenesis. ${ }^{27}$ In our study, RA was able to suppress proinflammatory cytokines. In particular, RA exerted an inhibitory effect on the IL-6/STAT3 pathway. Zhang et $\mathrm{al}^{28}$ also demonstrated that RA was able to inhibit proliferation and induce apoptosis in HSC-T6 mainly due to inhibition of phosphorylation in the STAT3 pathway. However, the function of RA in the proliferation of gastric cancer has not been investigated in previous studies.

The inflammatory response may also promote tumorigenesis via regulation of tumor-suppressive miRNAs. ${ }^{29}$ miRNAs are small RNAs, which have an important role in many physiological and pathological processes, including tumorigenesis and tumor suppression. ${ }^{30}$ Many studies have reported that abnormal miRNA expression is involved in a variety of tumors. ${ }^{31}$ In our study, miR-155, miR-146a, and miR-223, which are related to the inflammatory response, were suppressed by RA. miR-155 is an oncogenic miRNA, and has been found to be overexpressed in several types of malignant solid tumors in humans, including lung cancer, carcinomas of the digestive system, and breast cancer. ${ }^{32,33}$ Our study demonstrated that RA could significantly inhibit expression of miRNA-155-5p. To identify whether miR-155 was the target of the IL-6/STAT3 pathway of the Warburg, 

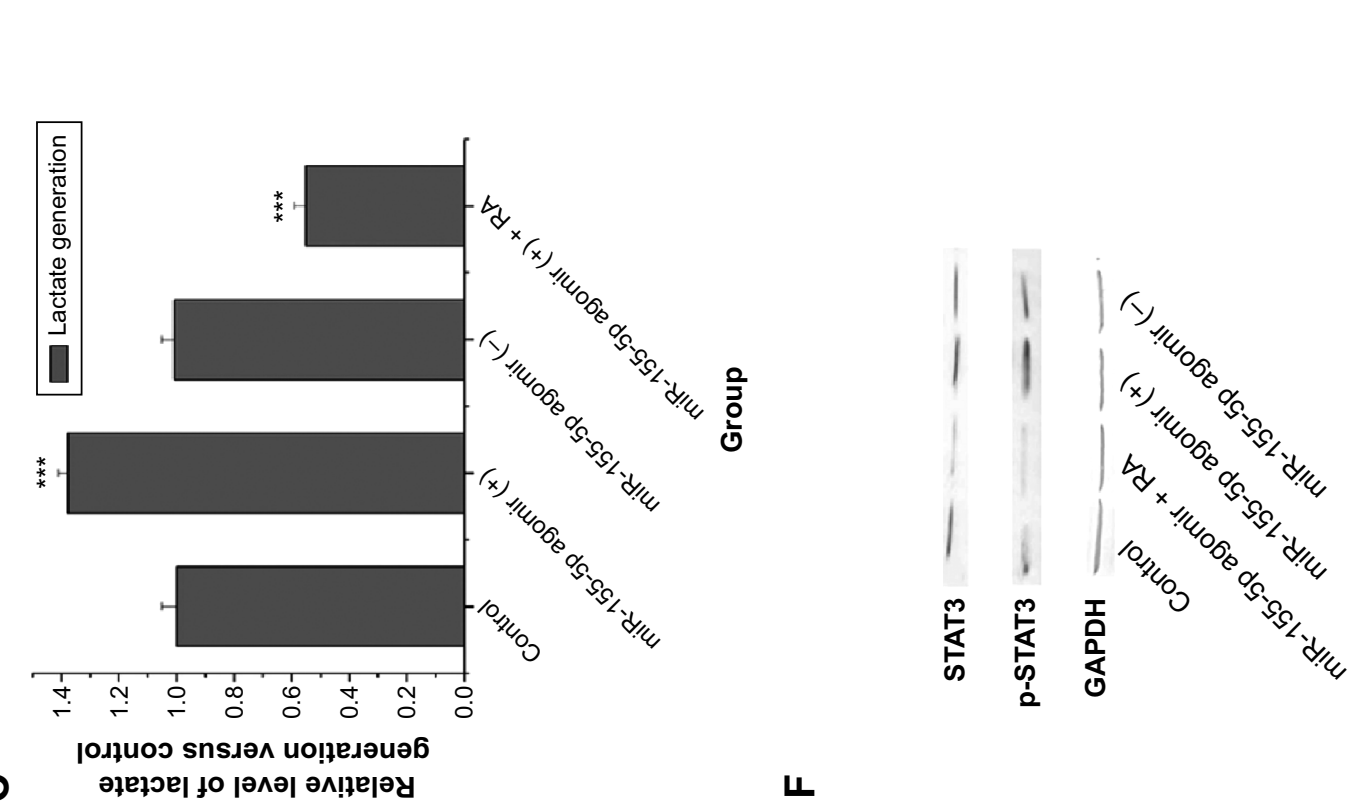

0

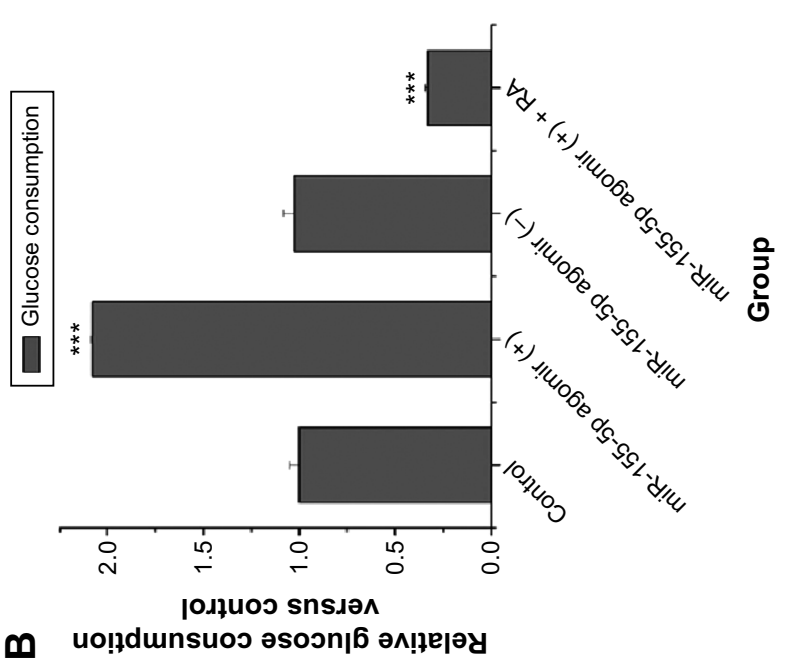

m uo!̣dunsuoว әsоэn|6 әк!?е|әу

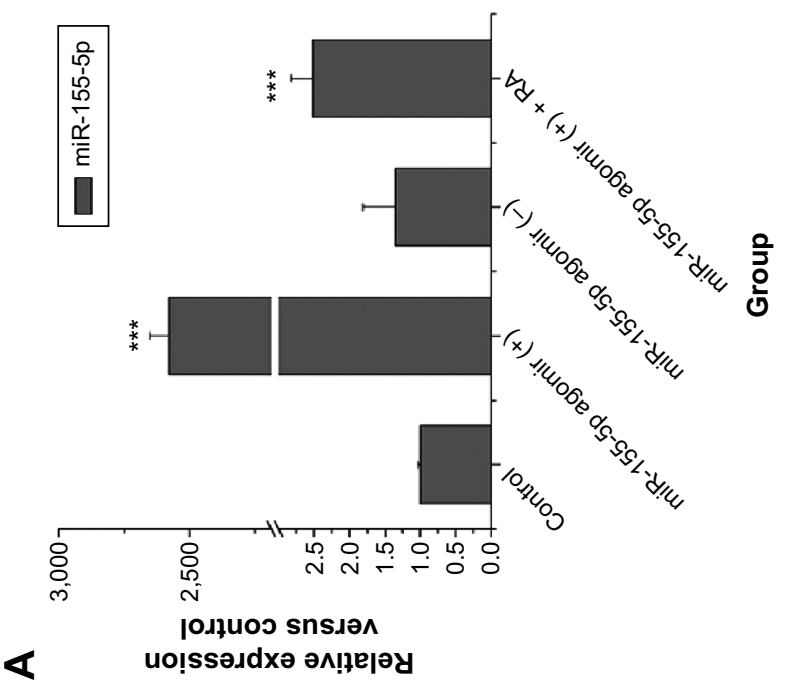

山

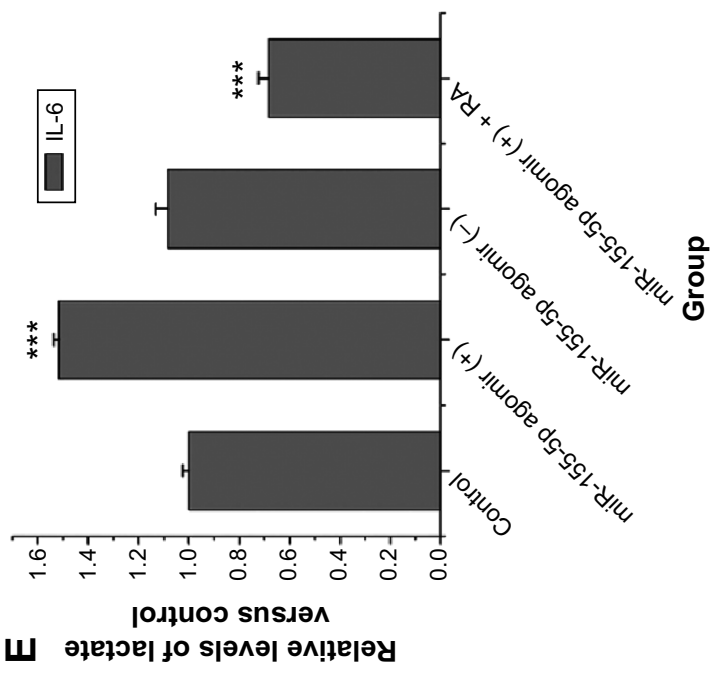

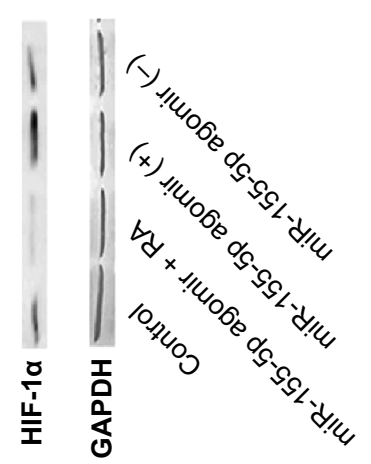




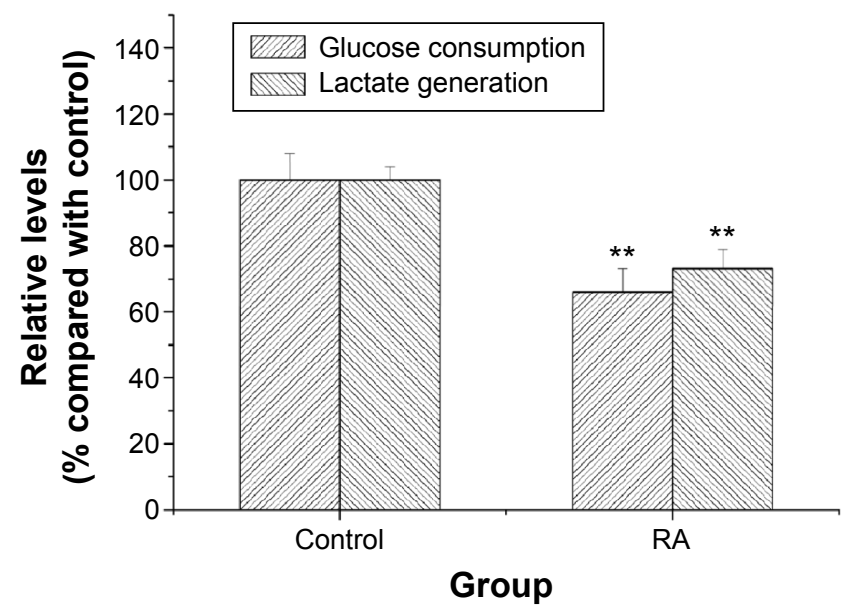

Figure 5 Effect of RA on levels of glucose consumption and lactate generation in vivo. The data are presented as the mean \pm standard deviation. Significant differences from control are indicated by $* * P<0.01$.

Abbreviation: RA, rosmarinic acid.

miRNA-155 was overexpressed by agomir. We then found that RA demonstrated an anti-Warburg effect, and dramatically inhibited the IL-6/STAT3 pathway.

The limitation of this study is that we only investigated the anti-Warburg effect of RA in a mouse model of gastric carcinoma, and not in other animals, such as rats and rabbits. This shortcoming may prevent use of RA in human clinical trials in the immediate future. Therefore, in upcoming studies, we will investigate the function of RA in gastric carcinoma in other animal species and use bioinformatics methods to confirm the present results.

In conclusion, our present study provides direct evidence that RA may be a candidate agent for suppression of the Warburg effect. RA has the ability to suppress glucose uptake and lactate production, and inhibits expression of HIF-1 $\alpha$, a transcription factor that affects the glycolytic pathway. Recent studies identified that inflammation promotes the Warburg effect in cancer cells. As expected, RA inhibited proinflammatory cytokines and miRNAs related to inflammation, suggesting that RA may suppress the Warburg effect via an inflammatory pathway, such as the IL-6/STAT3 pathway. miR-155 is considered to be an important mediator in the relationship between inflammation and tumorigenesis. We further showed that miR-155 was the target gene for regulating the Warburg effect by inactivating the IL-6/STAT3 pathway. Moreover, we found that RA suppressed the Warburg effect in vivo. Therefore, RA might be a potential therapeutic agent to suppress the Warburg effect in gastric carcinoma.

\section{Disclosure}

The authors report no conflicts of interest in this work.

\section{References}

1. Sawayama H, Ishimoto T, Sugihara H, et al. Clinical impact of the Warburg effect in gastrointestinal cancer (review). Int J Oncol. 2014; 45(4):1345-1354.

2. Simonnet H, Alazard N, Pfeiffer K, et al. Low mitochondrial respiratory chain content correlates with tumor aggressiveness in renal cell carcinoma. Carcinogenesis. 2002;23(5):759-768.

3. Moll UM, Schramm LM. p53 - an acrobat in tumorigenesis. Crit Rev Oral Biol Med. 1998;9(1):23-37.

4. Wang GL, Semenza GL. General involvement of hypoxia-inducible factor 1 in transcriptional response to hypoxia. Proc Natl Acad Sci U S A. 1993;90(9):4304-4308.

5. Semenza GL. Targeting HIF-1 for cancer therapy. Nat Rev Cancer. 2003;3(10):721-732.

6. Miller AH, Maletic V, Raison CL. Inflammation and its discontents: the role of cytokines in the pathophysiology of major depression. Biol Psychiatry. 2009;65(9):732-741.

7. Baiula M, Bedini A, Baldi J, Cavet ME, Govoni P, Spampinato S. Mapracorat, a selective glucocorticoid receptor agonist, causes apoptosis of eosinophils infiltrating the conjunctiva in late-phase experimental ocular allergy. Drug Des Devel Ther. 2014;8: 745-757.

8. Aggarwal BB, Vijayalekshmi RV, Sung B. Targeting inflammatory pathways for prevention and therapy of cancer: short-term friend, long-term foe. Clin Cancer Res. 2009;15(2):425-430.

9. Grivennikov SI, Greten FR, Karin M. Immunity, inflammation, and cancer. Cell. 2010;140(6):883-899.

10. Morgan MJ, Liu ZG. Crosstalk of reactive oxygen species and NFkappaB signaling. Cell Res. 2011;21(1):103-115.

11. Hanahan D, Weinberg RA. Hallmarks of cancer: the next generation. Cell. 2011;144(5):646-674.

12. Vaughan RA, Garcia-Smith R, Dorsey J, Griffith JK, Bisoffi M, Trujillo KA. Tumor necrosis factor alpha induces Warburg-like metabolism and is reversed by anti-inflammatory curcumin in breast epithelial cells. Int J Cancer. 2013;133(10):2504-2510.

13. Sahu A, Rawal N, Pangburn MK. Inhibition of complement by covalent attachment of rosmarinic acid to activated C3b. Biochem Pharmacol. 1999;57(12):1439-1446.

14. Xu Y, Jiang Z, Ji G, Liu J. Inhibition of bone metastasis from breast carcinoma by rosmarinic acid. Planta Med. 2010;76(10): 956-962.

15. Body JJ. Effectiveness and cost of bisphosphonate therapy in tumor bone disease. Cancer. 2003;97(3 Suppl):859-865.

16. Wu HP, Wang HL, Zhang Z, et al. [Analyze on the component of rosmarinic acid in Prunella vulgaris and its effect on alpha-glycosidase activity]. Zhong Yao Cai. 2011;34(11):1712-1715. Chinese.

17. Qiao S, Li W, Tsubouchi R, et al. Rosmarinic acid inhibits the formation of reactive oxygen and nitrogen species in RAW264.7 macrophages. Free Radic Res. 2005;39(9):995-1003.

18. Israf DA, Khaizurin TA, Syahida A, Lajis NH, Khozirah S. Cardamonin inhibits COX and iNOS expression via inhibition of p65NF-kappaB nuclear translocation and Ikappa-B phosphorylation in RAW 264.7 macrophage cells. Mol Immunol. 2007;44(5):673-679.

19. Shen K, Liang Q, Xu K, et al. MiR-139 inhibits invasion and metastasis of colorectal cancer by targeting the type I insulin-like growth factor receptor. Biochem Pharmacol. 2012;84(3):320-330.

20. Warburg O, Wind F, Negelein E. The metabolism of tumors in the body. J Gen Physiol. 1927;8(6):519-530.

21. Marusawa H, Jenkins BJ. Inflammation and gastrointestinal cancer: an overview. Cancer Lett. 2014;345(2):153-156.

22. McGirt LY, Adams CM, Baerenwald DA, Zwerner JP, Zic JA, Eischen CM. miR-223 regulates cell growth and targets proto-oncogenes in mycosis fungoides/cutaneous T-cell lymphoma. J Invest Dermatol. 2014;134(4):1101-1107.

23. Yuan CX, Zhou ZW, Yang YX, et al. Inhibition of mitotic aurora kinase a by alisertib induces apoptosis and autophagy of human gastric cancer AGS and NCI-N78 cells. Drug Des Devel Ther. 2015;9:487-508. 
24. Hsu PP, Sabatini DM. Cancer cell metabolism: Warburg and beyond. Cell. 2008;134(5):703-707.

25. Madhok BM, Yeluri S, Perry SL, Hughes TA, Jayne DG. Targeting glucose metabolism: an emerging concept for anticancer therapy. $\mathrm{Am}$ J Clin Oncol. 2011;34(6):628-635.

26. Gordo J, Maximo P, Cabrita E, et al. Thymus mastichina: chemical constituents and their anti-cancer activity. Nat Prod Commun. 2012;7(1):1491-1494.

27. Rosen DG, Mercado-Uribe I, Yang G, et al. The role of constitutively active signal transducer and activator of transcription 3 in ovarian tumorigenesis and prognosis. Cancer. 2006;107(11):2730-2740.

28. Zhang JJ, Wang YL, Feng XB, Song XD, Liu WB. Rosmarinic acid inhibits proliferation and induces apoptosis of hepatic stellate cells. Biol Pharm Bull. 2011;34(3):343-348.

29. Iliopoulos D, Hirsch HA, Struhl K. An epigenetic switch involving NF-kappaB, Lin28, Let-7 MicroRNA, and IL6 links inflammation to cell transformation. Cell. 2009;139(4):693-706.
30. Yang $\mathrm{Y}$, Peng W, Tang T, et al. MicroRNAs as promising biomarkers for tumor-staging: evaluation of MiR21 MiR155 MiR29a and MiR92a in predicting tumor stage of rectal cancer. Asian Pac J Cancer Prev. 2014; 15(13):5175-5180.

31. Chiang Y, Song Y, Wang Z, et al. microRNA-192, -194 and -215 are frequently downregulated in colorectal cancer. Exp Ther Med. 2012; 3(3):560-566.

32. Liu J, Gao J, Du Y, et al. Combination of plasma microRNAs with serum CA19-9 for early detection of pancreatic cancer. Int $J$ Cancer. 2012;131(3):683-691.

33. Kim YW, Kim EY, Jeon D, et al. Differential microRNA expression signatures and cell type-specific association with Taxol resistance in ovarian cancer cells. Drug Des Devel Ther. 2014;8:293-314.

\section{Publish your work in this journal}

Drug Design, Development and Therapy is an international, peerreviewed open-access journal that spans the spectrum of drug design and development through to clinical applications. Clinical outcomes, patient safety, and programs for the development and effective, safe, and sustained use of medicines are a feature of the journal, which has also been accepted for indexing on PubMed Central. The manuscript management system is completely online and includes a very quick and fair peer-review system, which is all easy to use. Visit http://www.dovepress.com/testimonials.php to read real quotes from published authors.

Submit your manuscript here: http://www.dovepress.com/drug-design-development-and-therapy-journal 